\title{
A potentiometric biosensor for rapid on-site disease diagnostics
}

\author{
Alexey Tarasov, ${ }^{1, *, \S}$ Darren W. Gray, ${ }^{2, *}$ Meng-Yen Tsai,,${ }^{1, *}$ Niall Shields, ${ }^{2, *}$ Armelle Montrose, ${ }^{3}$ \\ Niamh Creedon, ${ }^{3}$ Pierre Lovera, ${ }^{3}$ Alan O'Riordan, ${ }^{3}$ Mark H. Mooney, ${ }^{2}$ Eric M. Vogel ${ }^{1}$
}
${ }^{1}$ School of Materials Science and Engineering, Georgia Institute of Technology, Atlanta, GA 30332, USA
${ }^{2}$ Institute for Global Food Security, School of Biological Sciences, Queen's University Belfast, Belfast, Northern Ireland, BT9 5AG, United Kingdom
${ }^{3}$ Nanotechnology Group, Tyndall National Institute, University College Cork, Cork, T12R5CP, Ireland
*These authors contributed equally to this work
${ }^{\S}$ Corresponding author: alexey.tarasov@mse.gatech.edu

\begin{abstract}
Quantitative point-of-care (POC) devices are the next generation for serological disease diagnosis. Whilst pathogen serology is typically performed by centralized laboratories using Enzyme-Linked ImmunoSorbent Assay (ELISA), faster on-site diagnosis would infer improved disease management and treatment decisions. Using the model pathogen Bovine Herpes Virus-1 (BHV-1) this study employs an extended-gate field-effect transistor (FET) for direct potentiometric serological diagnosis. BHV-1 is a major viral pathogen of Bovine Respiratory Disease (BRD), the leading cause of economic loss (\$2 billion annually in the US only) to the cattle and dairy industry. To demonstrate the sensor capabilities as a diagnostic tool, BHV-1 viral protein $\mathrm{gE}$ was expressed and immobilized on the sensor surface to serve as a capture antigen for a BHV-1-specific antibody (anti-gE), produced in cattle in response to viral infection. The gE-
\end{abstract}


coated immunosensor was shown to be highly sensitive and selective to anti-gE present in commercially available anti-BHV-1 antiserum and in real serum samples from cattle with results being in excellent agreement with Surface Plasmon Resonance (SPR) and ELISA. The FET sensor is significantly faster than ELISA ( $<10 \mathrm{~min})$, a crucial factor for successful disease intervention. This sensor technology is versatile, amenable to multiplexing, easily integrated to POC devices, and has the potential to impact a wide range of human and animal diseases.

Keywords: field-effect transistors, potentiometric biosensors, immunosensors, serological diagnostics, Bovine Respiratory Disease, Bovine Herpes Virus-1

\section{Introduction}

The global population is predicted to increase to 9.3 billion by 2050 and food production will need to double to meet demand. With most suitable land already in use, this increase will need to come from greater efficiency facilitated by improved technology. At least $20 \%$ of animal-based food production is lost by infectious diseases and there is an overriding need to prevent and control animal disease. Due to advantages of high throughput and relatively low cost relative to other techniques such as PCR or IHC, serological testing by ELISA is routinely employed for disease surveillance purposes, but is performed in dedicated laboratories leading to long sample turnaround times and delays in result reporting. The development of low-cost point-of-care (POC) diagnostic devices would provide significant benefits in reducing the impacts of animal disease by enabling rapid identification, isolation and treatment of infected animals. Bovine Respiratory Disease (BRD) is currently the leading cause of economic loss ( $\$ 2$ billion annually in the US (Snowder et al. 2007)) to the cattle and dairy industry through concomitant clinical disease, therapeutic treatment, reduced animal performance and mortality (Scott 1994; Thompson et al. 2006). The BRD complex is therefore an exciting and realistic prospect for the application 
of POC sensor devices capable of multiplexing detection and facilitating on-site assessment of animal disease prevalence.

To date, no commercial POC devices exist for the serological assessment of pathogen related responses that can yield rapid quantifiable results with performance comparable to ELISA. Amongst available sensor technologies, potentiometric biosensors based on ion-sensitive field-effect transistors (ISFETs)(Bergveld 2003) are most promising due to their high sensitivity, rapid electronic readout, and compatibility with mainstream CMOS technology. The sensing principle relies on the binding of charged species (e.g. antibodies) to antigen immobilized on the sensor surface, modifying surface potential and hence the channel current inside the FET. Recently, many different material systems have been explored as potential alternatives for nextgeneration nanoelectronic sensors, including silicon nanowires (Si NWs) (Bedner et al. 2013; Chen et al. 2011; Cui et al. 2001; Knopfmacher et al. 2010; Knopfmacher et al. 2012; Livi et al. 2013; Noor and Krull 2014; Rajan et al. 2013; Stoop et al. 2015; Tarasov et al. 2012a; Tarasov et al. 2012b; Wipf et al. 2013), graphene (Fu et al. 2011; He et al. 2012; Zhan et al. 2014), carbon nanotubes (Tang et al. 2006), organic semiconductors (Bernards et al. 2008; Knopfmacher et al. 2014; Liao et al. 2015; Lin et al. 2011; Roberts et al. 2008), as well as $\mathrm{MoS}_{2}$-based FETs (Lee et al. 2014; Sarkar et al. 2014; Tarasov et al. 2014; Tarasov et al. 2015b; Tsai et al. 2015) and bandto-band tunneling FETs (Campbell et al. 2015; Sarkar et al. 2015). As a more robust and simple alternative, various configurations of extended-gate FETs have been studied (Guan et al. 2014; Rothberg et al. 2011), where the readout FET transducer is separated from the sensing chip and biological solution, thereby eliminating reliability issues (Fernandes et al. 2010). This significantly simplifies device fabrication and reduces costs as readout chips are reusable, whilst the sensing chips can be designed as low-cost disposables. Furthermore, the sensor surface can be 
engineered independent of the transducer to achieve stable selective functionalization, retaining the charge sensitivity of conventional or nanomaterial-based ISFETs (Dak et al. 2013; Tarasov et al. 2015a). Potentiometric sensors have not been thoroughly explored as possible diagnostic tools for monitoring immunological responses to infections and whilst several proof-of-concept studies have investigated model antigen-antibody interactions using various types of FETs, the majority of this previous work was performed in buffer systems, and not actual blood samples (Mu et al. 2015). Moreover, none of these studies attempted systematic comparison to established assay technologies such as SPR or ELISA to evaluate FET performance against current 'goldstandards' and assess true potential for real-world application.

Bovine Herpes Virus 1 (BHV-1) is a BRD viral pathogen (Ellis 2009) that induces respiratory tract damage and suppression of innate and adaptive immune responses leading to opportunistic bacterial (Confer 2009) and mycoplasma (Giovannini et al. 2013) infections. BHV1 virus is prevalent worldwide, however a number of countries have gained BHV-1 free status through the application of "test and cull" disease eradication programs (Raaperi et al. 2014) in combination with the use of efficacious marker vaccines based on a glycoprotein $\mathrm{E}(\mathrm{gE})$ negative BHV-1 strain (Strube et al. 1996). Differentiation of infected from vaccinated animals (DIVA) is achieved through the detection (using commercial companion anti-gE blocking ELISAs) of BHV-1 gE antibodies which are only present in animals exposed to wild-type virus (Wellenberg et al. 1998). Currently, disease surveillance schemes rely on centralized testing laboratories for the serological screening of bovine samples, with a minimum sample turnaround time of $72 \mathrm{~h}$ (including $19 \mathrm{~h}$ for $\mathrm{gE}$ blocking ELISA performance). BHV-1 exposed animals can also become latent carriers, with virus reactivation and shedding occurring during periods of stress e.g. animal mixing and transportation (Thiry et al. 1987). Therefore, rapid on-site identification of animals 
undergoing BHV-1 reactivation using quantitative POC devices (with DIVA capacity) would significantly reduce the risks of disease spread to BHV-1-free herds. The use of unpurified recombinant $\mathrm{gE}$ as a capture antigen in indirect ELISAs for serological diagnosis of BHV-1 has been shown to increase assay sensitivity enabling use of higher test sample dilutions (El-Kholy et al. 2013). Furthermore, purified recombinant viral capture antigen can improve test sensitivity by increasing the proportion of relevant antigenic material immobilized on test surfaces (Anderson et al. 2011). The development of a POC FET-based DIVA test for BHV-1 diagnosis using purified recombinant $\mathrm{gE}$ antigen would therefore allow for increased sample dilution overcoming Debye length limitations whilst maintaining assay sensitivity and facilitating rapid test result reporting.

Currently, eradication schemes based on vaccination and diagnostic disease surveillance using serological tests present an excellent opportunity to assess the potential of low-cost POC devices for rapid disease detection. The purpose of this study was to evaluate the application of recombinant BHV-1 $\mathrm{gE}$, expressed and purified using a Baculovirus expression system, as a capture antigen in the serological diagnosis of BHV-1 infection on low-cost extended-gate FET sensor platforms. A surface immobilization and regeneration protocol was developed for $\mathrm{gE}$ based on an SPR system, and selective binding of anti-gE to immobilized viral protein demonstrated. Haptoglobin (Hp) protein was used to assess potential non-specific binding issues due to a similar glycosylation pattern and molecular weight to that of recombinant $\mathrm{gE}$. Subsequently, gE was immobilized on an extended gate FET biosensor, which reported significant sensor responses to anti-gE with high selectivity, with FET results in excellent agreement to that found with SPR and commercial gE blocking ELISA. 


\section{Materials and methods}

\subsection{Reagents}

Bovine Herpesvirus-1 (BHV-1/IBR) antiserum (PAB-IBR, lot P110302-001) was purchased from Veterinary Medical Research \& Development (VMRD, Pullman, WA). Human haptoglobin (H3536, batch SLBJ3949V), rabbit anti-human haptoglobin (H8636, batch 072M4825V) and all other reagents unless stated were purchased from Sigma-Aldrich (Sigma-Aldrich, St. Louis, Missouri). Lyophilized anti-haptoglobin was reconstituted in distilled water as per manufacturer's instructions to yield a working stock concentration of $19.4 \mathrm{mg} / \mathrm{ml}$.

\subsection{Expression and Purification of BHV-1 glycoprotein gE recombinant antigen}

Recombinant BHV-1 gE was produced using the Bac-to-Bac (Life Technologies, Paisley, UK) Baculovirus expression system. A consensus amino acid sequence for the extracellular domain of BHV-1 gE (gE) was generated using NCBI deposited BHV-1 strain variants (Fig. S10) and codon optimized for production in Spodoptera frugiperda cells. Recombinant bacmid DNA containing BHV-1 gE (Bac+gE) was generated via TOPO cloning and homologous recombination with One Shot Max Efficiency DH5B and DH10Bac Escherichia coli strains respectively as per manufacturer's instructions. Recombinant Bac+gE DNA was purified from overnight culture of DH10Bac grown in LB broth containing $10 \mathrm{mg} / \mathrm{ml} \mathrm{kanamycin,} 50 \mathrm{mg} / \mathrm{ml}$ gentamicin, $10 \mathrm{mg} / \mathrm{ml}$ tetracycline). $25 \mathrm{ml}$ of overnight culture was centrifuged at $5,000 \mathrm{~g}$ at $4^{\circ} \mathrm{C}$ for $15 \mathrm{~min}$ and cell pellets were resuspended in $1 \mathrm{ml} 50 \mathrm{mM}$ Tris-Cl, $\mathrm{pH}$ 8.0, $10 \mathrm{mM}$ EDTA, 100 $\mu \mathrm{g} / \mathrm{ml}$ RNase A. $500 \mu \mathrm{l} 200 \mathrm{mM}$ NaOH, 1\% Sodium Dodecyl Sulphate (SDS) was added and following 5 min incubation $500 \mu \mathrm{l} 3.0 \mathrm{M}$ potassium acetate was added per aliquot. Following centrifugation at $12,000 \mathrm{~g}$ for $10 \mathrm{~min} 900 \mu \mathrm{l}$ of supernatant was removed, filtered to remove 
particulate, $630 \mu \mathrm{l}$ ice-cold isopropanol added and centrifuged at $16,000 \mathrm{~g}$ at $4{ }^{\circ} \mathrm{C}$ for $30 \mathrm{~min}$. The supernatant was removed and precipitated Bac+gE DNA was washed with $1 \mathrm{ml}$ ice cold $70 \%$ ethanol by centrifugation at $16,000 \mathrm{~g}$ at $4{ }^{\circ} \mathrm{C}$ for $15 \mathrm{~min}$. The supernatant was removed and Bac+gE DNA resuspended in $40 \mu \mathrm{l} 50 \mathrm{mM}$ Tris-Cl, $\mathrm{pH}$ 8.0, $10 \mathrm{mM}$ EDTA and stored at $-80{ }^{\circ} \mathrm{C}$. DNA concentration was determined using a NanoDrop 8000 (Thermo Scientific, UK). SF9 cells were transfected with $1 \mu \mathrm{g}$ Bac+gE DNA:2 $\mu$ l Escort IV transfection reagent using manufacturer's instructions and recombinant Baculovirus (P1 stock) was harvested when viability reached $50 \%$. P1 stock was amplified to high titer $\mathrm{P} 2$ and $\mathrm{Bac}+\mathrm{gE}$ titer was determined by plaque assay using established protocols (Brown and Faulkner 1978). SDS-PAGE was performed using 4-15\% TGX precast gels (Bio-Rad Laboratories, UK) to optimize expression conditions of $\mathrm{P} 2 \mathrm{Bac}+\mathrm{gE}$ stocks, with staining of High Five lysates and supernatant performed using colloidal coomassie (Anderson et al. 1995) and silver staining respectively (Bio-Rad silver stain kit).

High-Five suspension cultures, seeded at a density of $1.0 \times 10^{6}$ cells $/ \mathrm{ml}$ were infected with Bac-gE $\left(1.02 \times 10^{9} \mathrm{pfu} / \mathrm{ml}\right)$ at a multiplicity of infection of 3 plaque forming units per cell and harvested at 60 hours post infection. Harvested suspension cultures were centrifuged at 1,000 g for $5 \mathrm{~min}$ and the supernatant filtered using a $0.45 \mu \mathrm{m}$ PVDV Steritop Filter Unit (Millipore, UK). Recombinant histidine-tagged $\mathrm{gE}$ was purified using immobilized metal ion affinity chromatography (IMAC) using the AKTA Start purification system coupled to a Frac30 and operating under Unicorn Start 1.0 (GE Healthcare Life Sciences, UK). The mobile phase consisted of PBS, 200 mM KCl, $20 \%$ glycerol, pH 7.2 (buffer A) and PBS, $20 \mathrm{mM} \mathrm{KCl,} 20 \%$ glycerol, 500 mM imidazole, pH 7.2 (buffer B). A 5 ml HisTrap Excel column, pre-packed with $\mathrm{Ni}$-sepharose was equilibrated with 5 column volumes $100 \%$ buffer A at a flow rate of $5 \mathrm{ml} / \mathrm{min}$. 
Filtered High Five supernatant containing his-tagged $\mathrm{gE}$ was injected at a flow rate of $1 \mathrm{ml} / \mathrm{min}$ followed by washing with 15 column volumes $100 \%$ buffer A followed by 10 column volumes of $10 \%$ and $20 \%$ buffer $\mathrm{B}$ respectively at a flow rate of $5 \mathrm{ml} / \mathrm{min}$. gE was eluted with 10 column volumes of $50 \%$ buffer $\mathrm{B}$ respectively at a flow rate of $5 \mathrm{ml} / \mathrm{min}$. Purity of eluted proteins was assessed by SDS-PAGE (4-15 \% TGX gels with silver staining) and western blot.

\subsection{SDS-PAGE and western blot analysis of purified gE}

Identity and reactivity of purified recombinant $\mathrm{gE}$ was determined by SDS-PAGE and western blot. $4 \mu \mathrm{l}$ loading buffer was added to $20 \mu \mathrm{l}$ of protein complexes and heated for $5 \mathrm{~min}$ at $95{ }^{\circ} \mathrm{C}$. Samples were loaded onto individual wells on TGX 4-15\% precast gels (Bio-Rad). $10 \mu$ of Novex Sharp Pre-stained Ladder (LifeTechnologies) or ECL Dual View Marker (GE Healthcare Life Sciences) was added to empty lanes for gels designated for SDS-PAGE and western blot analysis respectively and electrophoresis for $50 \mathrm{~min}$ at $100 \mathrm{~V}$. Gels for SDS-PAGE analysis were stained with colloidal coomassie (Anderson et al. 1995) or Bio-Rad silver staining kit where appropriate. Gels selected for western blot were transferred electrophoretically onto PVDF membranes (GE Healthcare Life Sciences) using wet blotting at $10 \mathrm{~mA}$ overnight. The PVDF membrane was probed with 1:500 goat anti-BHV-1 antiserum in $20 \mathrm{mM}$ Tris $\mathrm{pH} 7.5,150 \mathrm{nM}$ $\mathrm{NaCl}, 0.1 \%$ Tween 20 for $2 \mathrm{~h}$ at room temperature. The PVDF membrane was washed with TBST and blocked with TBST containing 3\% BSA for $1 \mathrm{~h}$. After washing with TBST the membrane was probed with 1:20,000 horse radish peroxidase conjugated rabbit anti-goat IgG (Sigma Aldrich) for $2 \mathrm{~h}$ at room temperature. The membrane was washed thoroughly and chemiluminescence detection was performed on X-ray film using ECL-prime (GE Lifesciences, UK) following the manufacturer's instructions.

\subsection{Protein immobilization and measurements of antibody binding on Biacore CM5 chips}


$\mathrm{pH}$ scouting was performed to determine buffer conditions for maximal deposition of BHV-1 $\mathrm{gE}$ at chip surfaces. $50 \mu \mathrm{g} / \mathrm{ml} \mathrm{BHV-1} \mathrm{gE}$ (or Human Haptoglobin (Hp) for control studies) was prepared in $10 \mathrm{mM}$ sodium acetate $\mathrm{pH} \mathrm{3.8,} 4.0$ and 4.5 from $0.4 \mathrm{mg} / \mathrm{ml}$ working stock. $15 \mu \mathrm{l}$ of BHV-1 $\mathrm{gE}$ (or Hp) prepared at varying $\mathrm{pH}$ in buffer was injected at a flow rate of $5 \mu \mathrm{l} / \mathrm{min}$. Excess ligand was removed with a $10 \mu \mathrm{l}$ injection of $200 \mathrm{mM} \mathrm{NaOH}$ at a flow rate of $20 \mu \mathrm{l} / \mathrm{min}$. BHV-1 gE (or Hp) was covalently immobilized to the surface of CM5 classic (Biacore Q) chips using Biacore amine coupling kit according to the manufacturer's instructions. Immobilization was performed using the IFC microfluidic system. Between infections $1 \times$ HBS-EP was used as a running buffer for removal of unbound coupling reagents. Briefly, EDC and NHS, prepared according to the amine coupling kit in separate vials $(750 \mathrm{mg}$ EDC and $115 \mathrm{mg}$ NHS each resuspended in $10 \mathrm{ml} 0.22 \mu \mathrm{m}$ filtered dd- $\mathrm{H}_{2} \mathrm{O}$ ), were mixed 1:1 via the Biacore Q. $35 \mu 1$ of 1:1 EDC:NHS mixture was injected at a flow rate of $5 \mu 1 / \mathrm{min}$ to activate the CM5 chip surface. 50 $\mu \mathrm{l}$ of $50 \mu \mathrm{g} / \mathrm{ml} \mathrm{BHV}-1 \mathrm{gE}$ (or $\mathrm{Hp}$ ) (prepared in $10 \mathrm{mM}$ sodium acetate $\mathrm{pH} 4.0$ ) was conjugated to the activated chip at a flow rate of $5 \mu \mathrm{l} / \mathrm{min}$. The chip surface was deactivated by injection of 30 $\mu \mathrm{l}$ ethanolamine- $\mathrm{HCl} \mathrm{pH} 8.5$ at a flow rate of $5 \mu \mathrm{l} / \mathrm{min}$. Unbound BHV-1 $\mathrm{gE}$ (or Hp) was removed with $510 \mu \mathrm{l}$ injections of $10 \mathrm{mM}$ glycine- $\mathrm{HCl} \mathrm{pH} 1.5$ and $210 \mu 1$ injections of $10 \mathrm{mM}$ glycine- $\mathrm{HCl} \mathrm{pH} 2.0$ at a flow rate of $20 \mu \mathrm{l} / \mathrm{min}$. To evaluate the binding, $15 \mu \mathrm{l}$ of 1:10,000, $1: 1,000,1: 500,1: 200,1: 100,1: 50,1: 20$ and 1:10 dilutions of anti-BHV-1 working stock, prepared in $1 \times$ HBS-EP, were injected at a flow rate of $5 \mu \mathrm{l} / \mathrm{min}$ onto a CM5 chip immobilized with $\mathrm{gE}$ (or Hp). Following antibody binding the chip surface was regenerated with $10 \mu \mathrm{l}$ injections of $10 \mathrm{mM}$ Glycine- $\mathrm{HCl} \mathrm{pH} 2.0$ at a flow rate of $20 \mu \mathrm{l} / \mathrm{min}$ to remove Anti-BHV-1 bound to immobilized BHV-1 gE (or Hp). To investigate antibody response specificity $15 \mu \mathrm{l}$ of different concentrations of anti-Hp, prepared in 1 x HBS-EP, were injected using the same 
parameters as $\mathrm{gE}$ onto CM5 chip immobilized with $\mathrm{gE}$ (or Hp). Regeneration was performed using $10 \mu \mathrm{l}$ injection of $22 \mathrm{mM} \mathrm{NaOH}$ at a flow rate of $20 \mu \mathrm{l} / \mathrm{min}$.

\subsection{Protein immobilization and measurements of antibody binding on Sierra SPR chips}

BHV-1 gE was covalently immobilized to the surface of SPR chips using a Sierra Sensors SPR 2 instrument. These SPR chips have a SAM of mercaptohexadecanoic acid, similar to the FET chips. Briefly, a freshly mixed EDC $(750 \mathrm{mg} / \mathrm{ml})$ and NHS $(115 \mathrm{mg} / \mathrm{ml})$ were mixed 1:1 and injected to activate the surface $(175 \mu 1$, at a flow rate of $25 \mu 1 / \mathrm{min})$. Then, a $\mathrm{gE}$ solution was injected to immobilize the proteins on the activated surface $(200 \mu \mathrm{l} ; 50 \mu \mathrm{g} / \mathrm{ml}$ in $10 \mathrm{mM}$ sodium acetate $\mathrm{pH} 4.0$; flow rate $25 \mu \mathrm{l} / \mathrm{min}$ ). The chip surface was then deactivated with injection of 150 $\mu \mathrm{l}$ of ethanolamine- $\mathrm{HCl} \mathrm{pH} 8.5$ at a flow rate of $25 \mu \mathrm{l} / \mathrm{min}$. Unbound $\mathrm{BHV}-1 \mathrm{gE}$ was removed with $515 \mu \mathrm{l}$ injections of $10 \mathrm{mM}$ glycine- $\mathrm{HCl} \mathrm{pH} 1.5$ and $2.015 \mu \mathrm{l}$ injections of $10 \mathrm{mM}$ glycine$\mathrm{HCl} \mathrm{pH} 2.0$ at a flow rate of $25 \mu 1 / \mathrm{min}$.

$5 \mu \mathrm{l}$ of $1: 10,000,1: 1,000,1: 500,1: 200,1: 100,1: 50,1: 20$ and $1: 10$ dilutions of anti-BHV1 working stock, prepared in 1 x HBS-EP, were injected at a flow rate of $25 \mu 1 / \mathrm{min}$ onto a SAM chip with immobilized gE (or Hp). Following antibody binding the chip surface was regenerated with $5 \mu \mathrm{l}$ injections of $10 \mathrm{mM}$ glycine- $\mathrm{HCl} \mathrm{pH} 2.0$ at a flow rate of $25 \mu \mathrm{l} / \mathrm{min}$ to remove antiBHV-1 bound to immobilized BHV-1 gE (or Hp).

\subsection{Fabrication of EGFET biosensors and electrical measurements}

A silicon wafer with $260 \mathrm{~nm}$ oxide was used as a substrate for the sensing chip. Note that any other insulating substrate can be used, in principle, including plastic or paper. A thin gold film $(100 \mathrm{~nm})$ was deposited by e-beam evaporation through a shadow mask to form 4 individual gold strips. A home-made polydimethylsiloxane (PDMS) microfluidics cell was mounted on the chip 
for the functionalization and measurements. The area of the strips in contact with the liquid was 2 $\mathrm{mm} \times 3 \mathrm{~mm}$, defined by the liquid cell dimensions. Two of 4 strips were coated with $\mathrm{gE}$, and the remaining 2 were coated with $\mathrm{Hp}$, closely following the immobilization protocol developed on Biacore. Each individual gold strip was electrically connected to the gate terminal of a commercial MOSFET. Note that any type of transistor can be used for the readout, including nanomaterial-based FETs. A flow-through reference electrode (Ag/AgCl; Microelectrodes Inc.) was placed in the tubing close to the sensor in order to gate the transistor through the liquid during the measurements. The electrical measurements were performed using a semiconductor parameter analyzer (Keithley 4200-SCS and HP 4145A).

\subsection{Protein immobilization and measurements of antibody binding on EGFET chips}

The gold surface of the sensors was cleaned by a 20 min UV/ozone treatment. The samples were then immersed in a solution of 16-mercaptohexadecanoic acid (MHA, Sigma Aldrich, 5 mM in ethanol) and left overnight (approx. 18 hours) to create a self-assembled monolayer (SAM) with functional carboxyl $(\mathrm{COOH})$ groups. The samples were rinsed with ethanol and DI water. Then, the microfluidic chip was placed on the sample equipped with 4 different channels to selectively functionalize the active and the control channels. The sample was flushed with HBS-EP buffer before each functionalization step. A fresh NHS/EDC mixture was prepared in DI water as described above, and injected into the microfluidic cell and left inside to activate the surface for approx. $30 \mathrm{~min}$. Using 2 out of 4 channels, BHV-1 gE protein solution $(50 \mu \mathrm{g} / \mathrm{ml}$, prepared in 10 $\mathrm{mM}$ sodium acetate buffer, $\mathrm{pH} 4.0$ ) was delivered to the activated chip. In the remaining 2 channels, a solution of the control protein $\mathrm{Hp}(50 \mu \mathrm{g} / \mathrm{ml}, 10 \mathrm{mM}$ sodium acetate, $\mathrm{pH} 4.0)$ was injected. The reaction time was $30 \mathrm{~min}$ for both proteins. The chip surface was deactivated by injection of ethanolamine- $\mathrm{HCl} \mathrm{pH} 8.5$ for $30 \mathrm{~min}$. Measurements were performed in different 
dilutions of anti-BHV-1 antiserum or anti-Hp (control), sequentially from lowest to highest concentration. The reaction time with antiserum or anti-Hp was $10 \mathrm{~min}$, the volume was approx. $300 \mu \mathrm{l}$ for each dilution. All dilutions were prepared in $10 \mathrm{mM}$ HBS-EP buffer $\mathrm{pH}$ 7.4. The samples were regenerated with $300 \mu \mathrm{l}$ of $10 \mathrm{mM}$ glycine $\mathrm{pH} 2$ for $1 \mathrm{~min}$.

\subsection{BHV-1 gE blocking ELISA}

Commercial gE blocking ELISA (IDEXX, Laboratories, Maine, USA) was performed as per the manufacturer's instructions. Briefly, $100 \mu \mathrm{l}$ of 1:10,000, 1:1,000, 1:500, 1:200, 1:100, 1:50, 1:20 and 1:10 anti-BHV-1 working stock dilutions, prepared in dilution buffer were added to duplicate wells on a 96 well plate coated with BHV-1 antigen. Positive and negative control samples (1:2 in dilution buffer) were also added for test validation. Following $18 \mathrm{hr}$ incubation at $22{ }^{\circ} \mathrm{C}$ the wells containing serum were washed 5 times with $300 \mu \mathrm{l}$ wash buffer and $100 \mu$ conjugate (antigE HRP MAb) added for $30 \mathrm{~min}$ at $22{ }^{\circ} \mathrm{C}$. The plate was washed 5 times with $300 \mu \mathrm{l}$ wash buffer, $100 \mu \mathrm{TMB}$ substrate added and incubated for $15 \mathrm{~min}$ at $22{ }^{\circ} \mathrm{C} .100 \mu \mathrm{l}$ of stop solution was added to stop the reaction and absorbance was measured at $650 \mathrm{~nm}$. 


\section{Results}

\subsection{Production and purification of BHV-1 glycoprotein gE capture antigen}

Recombinant BHV-1 gE protein for sensor immobilization was expressed using the Baculovirus expression system (as illustrated in Fig. 1a). Fig. 1b illustrates that truncated BHV-1 gE was successfully expressed and secreted in High Five insect cells. During expression optimization a major band at $59 \mathrm{kDa}$ was evident at 24-96 h post-infection (p.i.), with increasing levels of cellular derived protein contaminants and protease degradation evident from $72 \mathrm{~h}$ (data not shown). A multiplicity of infection of 3 pfu/cell and harvesting at $66 \mathrm{~h}$ p.i. was selected to maximize gE secretion enabling one-step purification under native conditions by IMAC (Fig. 1b). Antigenicity of the consensus gE sequence was evidenced by positive reaction with antiBHV-1 antiserum (Fig. 1c). IMAC fractions containing $\mathrm{gE}$ were pooled and concentrated to 1 $\mathrm{mg} / \mathrm{ml}$ for sensor immobilization. Purity of the major band at $59 \mathrm{kDa}$ was calculated at $93 \%$ on SDS-PAGE gels using densitometry analysis. SDS-PAGE and western blot analysis (Fig. 1d and 1e respectively) of purified gE stocks revealed a number of immuno-reactive bands with antiBHV-1 antiserum: a major band at $59 \mathrm{kDa}$ with minor bands at 37, 43, and $84 \mathrm{kDa}$ on SDS-Page and 41, 84 and $119 \mathrm{kDa}$ on western blot. Predicted gE molecular weight (MW) based on amino acid sequence with removal of signal peptide is $41.7 \mathrm{kDa}$ with the $59 \mathrm{kDa}$ band corresponding to glycosylated gE. Minor bands are likely to originate from non-glycosylated gE (41-43 kDa) and dimers of gE (84 kDa) and glycosylated-gE (119 kDa).

\subsection{Fabrication of EGFET biosensor}


In parallel to protein expression, the potentiometric sensing device was developed. Fig. 2a shows a schematic of the device consisting of two parts: a sensor for signal generation (right) and a transducer for signal amplification and readout (left). The active sensing surface is a thin gold film $(100 \mathrm{~nm})$ evaporated on a $\mathrm{Si} / \mathrm{SiO}_{2}$ substrate. The gold film is in contact with the electrolyte and its surface potential depends on the amount of charged molecules attached to the surface. The gold surface can be chemically modified to achieve specific adsorption of certain biomolecules. There are several reasons for choosing a gold film: first, access to well-established thiol-based surface chemistry for self-assembly of linker molecules; second, the possibility of a direct comparison to commercial tools based on SPR, which also typically use a gold surface; and, third, excellent chemical stability even in harsh environments. To read out the potential changes on the gold surface, a commercial MOSFET was used as the readout transducer. The gold film acts as the so-called "extended gate" of the MOSFET, and is electrically connected to the gate terminal. $\mathrm{An} \mathrm{Ag} / \mathrm{AgCl}$ reference electrode was immersed in the solution to gate the transistor through the liquid. Different solutions were delivered to the surface using microfluidics. In Fig. 2b, a typical transfer curve of the liquid-gated extended-gate FET (EGFET) in buffer is shown. The drain current $I_{\mathrm{d}}$ can be modulated by sweeping the voltage at the reference electrode $V_{\text {ref. }}$ To enable different functionalization on one chip, a disposable sensing chip with 4 gold strips was used (Fig. 2c) -- each gold strip can be independently functionalized using a liquid cell (shown in Fig. 2d).

\subsection{Evaluation of FET sensor performance for the serological diagnosis of BHV-1}

Glycoprotein gE was covalently immobilized on the gold surface of the EGFET via amine coupling to the carboxyl groups of a self-assembled monolayer (SAM) of thiol linker molecules. 
For comparison, two different SPR chips were used in this study (Fig. 1a). A standard Biacore CM5 chip (carboxylated dextran covalently attached to a gold surface) was first used to develop the protein immobilization protocol and to evaluate the antibody binding. The advantage of the CM5 chips is a high binding capacity and response, as a result of high density of carboxyl groups present in the dextran matrix. To ensure the best possible comparison to the FET sensors which use carboxylated monothiol SAMs rather than a dense dextran matrix, SPR chips with a monothiol SAM (Sierra Sensors) were also prepared and compared to CM5 surfaces (Fig. S4d). Both SPR chips show qualitatively the same behavior, and yield results comparable to the FET sensor, with a higher response on dextran surfaces compared to SAM, attributed to a higher density of carboxyl groups. In the following, SPR data from SAM chips will be used for comparison with FET results, unless stated otherwise.

To evaluate EGFET sensor performance for the detection of BHV-1, the gE-coated gold strip was measured in different dilutions of anti-BHV-1 antiserum and Fig. 3a illustrates transfer curves shifting to the left with increasing antibody concentration. As a control for non-specific binding, a second gold strip was coated with human Hp, and measured in the same antiserum dilutions. The level of Hp protein immobilization was similar to that of $\mathrm{gE}$ as seen in Fig. S6a and Fig. S7a. The response of the active EGFET, defined as the gate voltage change relative to the value measured in buffer (i.e. response in buffer $=0 \mathrm{mV}$ ), taken at a drain current of $10 \mathrm{nA}$, is plotted versus antiserum dilution in Fig. 3b (squares), together with SPR responses (circles). Both sensors exhibit significant and very similar response even at antiserum dilutions as low as 1:1000. In contrast to the active sensor, the Hp-coated control does not show a clear trend with changing antiserum concentration (Fig. 3c). On FET and SPR sensors the observed response due to non-specific binding is small (Fig. 3d and Fig. S4b) compared to the specific response shown 
in Fig. 3b. To test the selectivity of the sensor, the active gE-coated gold strip was measured in different dilutions of $\mathrm{Hp}$ antibody (anti-Hp) (illustrated in Fig. 4). The EGFET only weakly responds to changes in anti-Hp concentration with minimal shift to the right (squares in Fig. $\mathbf{4 b}$ ) a similar weak response was observed with SPR (circles). These calibration results suggest that the expressed $\mathrm{gE}$ protein is functional on the sensor surface, and the EGFET sensor is highly sensitive and selective to anti-gE within the antiserum with very similar performance to SPR. As an additional control, the Hp-coated chip was also measured in different anti-Hp dilutions (Fig. S1). As expected, a strong response was observed, providing more evidence for the high specificity of the interactions studied here. The results are also consistent across different samples under the same modification and characterization conditions (Fig. S2).

The EGFET results were further verified using commercial gE ELISA kits (IDEXX). In Fig. 5, data from the gE-coated FET sensor (from Fig. 3b) is compared with ELISA absorption measurements, performed in the same dilutions of anti-BHV-1 antiserum. All test platforms (FET, SPR, ELISA) have a similar dynamic range and approach the limit of detection for anti-gE in sera dilutions $<2 \cdot 10^{-3}$. These results indicate that EGFET sensors can have a performance comparable to well established and optimized ELISA assays. In addition, the EGFET sensors provide results much faster than ELISA (<10 min vs $19 \mathrm{~h}$ ), making them very promising for rapid diagnostic applications.

The respective sensors were also tested in several dilutions of bovine serum samples. The results are presented in Fig. 6a for the EGFET sensor, and Fig. 6b for the SPR, with both sensors showing similar response (see Supplementary Material for raw data). Serum sample 1 is from a BHV-1 negative animal whilst sera samples 2 and 3 originate from BHV-1 positive animals. As expected, FET and SPR responses were higher for BHV-1 positive animals compared to negative 
animals, with increasing response (maintaining the same trend) with sera concentration (1:50, 1:20, 1:10). Furthermore, the sensor can be regenerated by removing bound antibodies with glycine without damaging the immobilized gE proteins (Fig. S2). Whilst the sensor part can also be designed as a single-use disposable, this feature can be a significant cost advantage enabling a single sensor to test multiple samples. Whilst these initial results are extremely promising, further work will be required to fully establish the FET sensors for disease monitoring purposes within populations.

\section{Discussion}

Currently there is no commercial low-cost point-of-care device for the serological diagnosis of pathogen related responses in blood that can yield rapid quantifiable results approaching the sensitivity of ELISA. FET based biosensors are particularly promising for user-friendly, rapid diagnosis required for effective on-site disease control. This is the first demonstration of a potentiometric sensor for diagnosis of animal diseases using blood. The endemic BRD virus BHV-1 was selected as a model pathogen for investigating FET sensor performance due to the high level of screening required for current and planned disease eradication programs. BHV-1

gE glycoprotein was engineered to allow for high level secretion and simple purification with minimal sample preprocessing via the removal of the intracellular and transmembrane regions as achieved with similar viral membrane proteins (Donofrio et al. 2006; Lehman et al. 1993). Produced $\mathrm{gE}$ was found to be suitable for diagnostic test development with antigenicity confirmed through positive reaction with anti-BHV-1 antiserum. By using identical sensing surface (gold) and protein immobilization protocols, SPR and FET sensor systems were evaluated and found to yield equivalent results for antibody binding to $\mathrm{gE}$ (in serum) and $\mathrm{Hp}$. Reduced Debye length and non-specific binding have traditionally been limiting factors for direct FET 
sensing in serum, requiring complicated sample pre-treatment (Stern et al. 2010) and reducing their potential for rapid POC testing. Compared to complicated desalting approaches (Chua et al. 2009), this study applied simple dilution in a fixed ionic strength buffer to increase Debye length and whilst signal response was low, a sigmoidal response was achieved for anti-BHV-1 binding to immobilized $\mathrm{gE}$ with comparable sensitivity and dynamic range to SPR and commercial ELISA. The application of a reference electrode with immobilized Hp glycoprotein allowed for the elimination of non-specific binding effects observed in SPR and FET sensors at higher serum dilutions $(1: 10-1: 20)$. Particularly interesting is the observation that in actual serum samples from BHV-1 positive or negative animals, the FET sensors gave results comparative to that achieved by SPR. These results are extremely promising and highlight that the application of FET sensors to serological disease diagnosis is transferable to a multitude of pathogenic microorganisms.

Unlike SPR, FET sensors do not require any optical components, are low-cost and portable. The use of extended-gate geometry rather than a conventional ISFET further simplifies device fabrication and reduces the cost of the sensor. Separating the readout transducer from the gold sensing surface has the following main advantages: 1. reliability issues can be avoided because the transducer is not in contact with the biological solution; 2. the transducer is reusable and can be developed independent of the sensor surface to optimize the signal-to-noise ratio (Bedner et al. 2014; Fu et al. 2013; Tarasov et al. 2011); 3. the sensing chip can be designed as an inexpensive disposable in a very simple fashion (chemically modified thin gold strips on an arbitrary low-cost substrate). Having several gold strips can potentially enable multiplexed detection of several analytes. The presented sensors can be easily manufactured at a large scale, resulting in very low cost per test. Compared to ELISA, the FET sensor is several orders of 
magnitude faster (<10 min POC vs. $>72$ hours for laboratory based testing), which is critical for the diagnosis of highly contagious diseases. Future work will focus on increasing the density of attachment sites and optimizing incubation times and other relevant assay parameters to further improve FET performance.

\section{Conclusions}

A novel potentiometric sensor based on extended-gate field-effect transistors in a dual-chip configuration has been presented. The sensor consists of a MOSFET transducer for signal amplification and a separate gold-coated chip for signal generation. The gold surface is brought in contact with liquid using microfluidics, and is electrically connected to the gate terminal of the MOSFET during the experiment. To demonstrate the sensor capabilities as a diagnostic tool, BHV-1 viral protein gE was expressed and immobilized on the gold surface to serve as a capture antigen for a BHV-1-specific antibody (anti-gE), produced in cattle in response to viral infection. The gE-coated immunosensor was shown to be highly sensitive and selective to anti-gE present in commercially available anti-BHV-1 antiserum and in real serum samples from cattle with results being in excellent agreement with SPR and ELISA. The presented transistor-based sensor platform not only achieves equivalent performance to SPR and ELISA, but also offers several distinct advantages over these techniques. In particular, it does not require expensive optical components, provides results much faster than ELISA ( $<10 \mathrm{~min})$ and can be used in the field without the need for central labs, reducing critical delays. Furthermore, the sensor is easy-to-use, label-free, has a disposable sensing part, and can be extended to other diseases for multiparameter testing. We anticipate a strong impact of this work on the on-site diagnostics and prevention of animal diseases, leading to significant cost reduction for the agricultural industry. 


\section{Acknowledgements}

This work was supported by: the National Science Foundation (NSF, through CBET Award \#1264705); the Swiss National Science Foundation (SNSF) (Grants \#P2BSP2_148636, \#P300P2_158502, to A.T.); Science Foundation Ireland (SFI) under US-Ireland Programme (SFI12/US/I2476); the Department for Employment and Learning, Northern Ireland (DELNI, Grant\# USI 039); and the Center for Low Energy Systems Technology, one of six centers supported by the STARnet phase of the Focus Center Research Program, a Semiconductor Research Corporation program sponsored by MARCO and DARPA.

\section{Author contributions}

A.T. and M.-Y.T. designed, fabricated and characterized the FET-based sensors. D.W.G. and N.S. expressed, purified and characterized the proteins. A.T., M.-Y.T., D.W.G., N.S., A.M., N.C., P.L. designed and performed the biosensing experiments, analyzed and interpreted results. A.T. and D.W.G. wrote the manuscript. A.O., M.H.M., E.M.V. conceived and supervised the work. All authors read and approved the manuscript. 
(a)

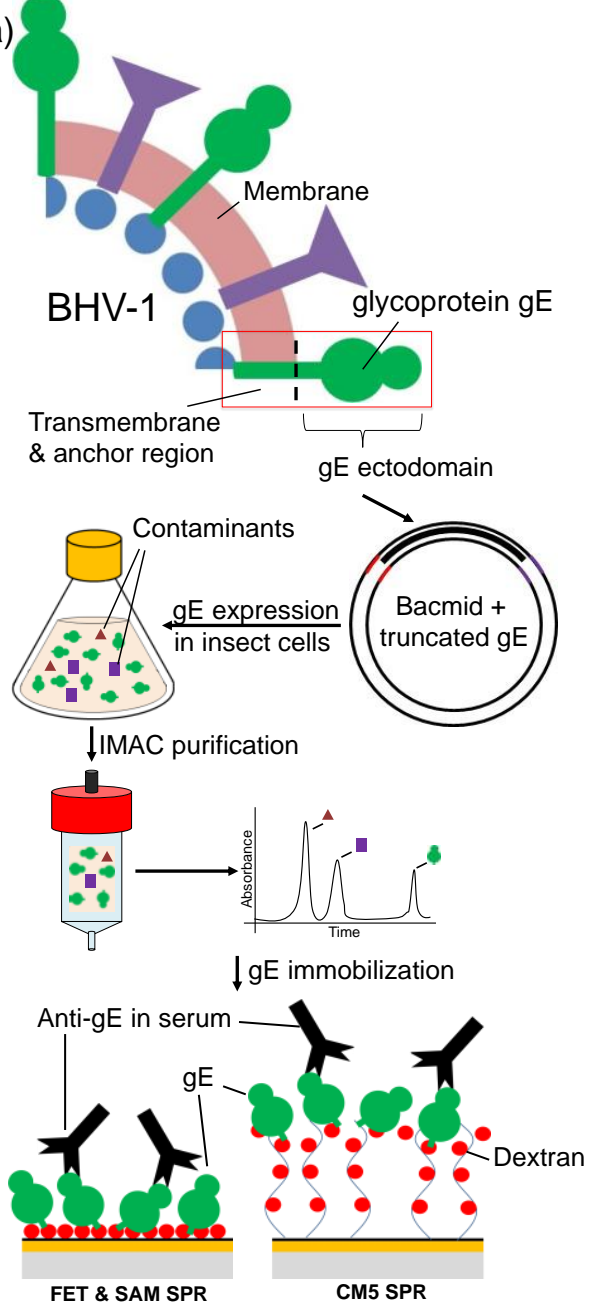

(b) (c)

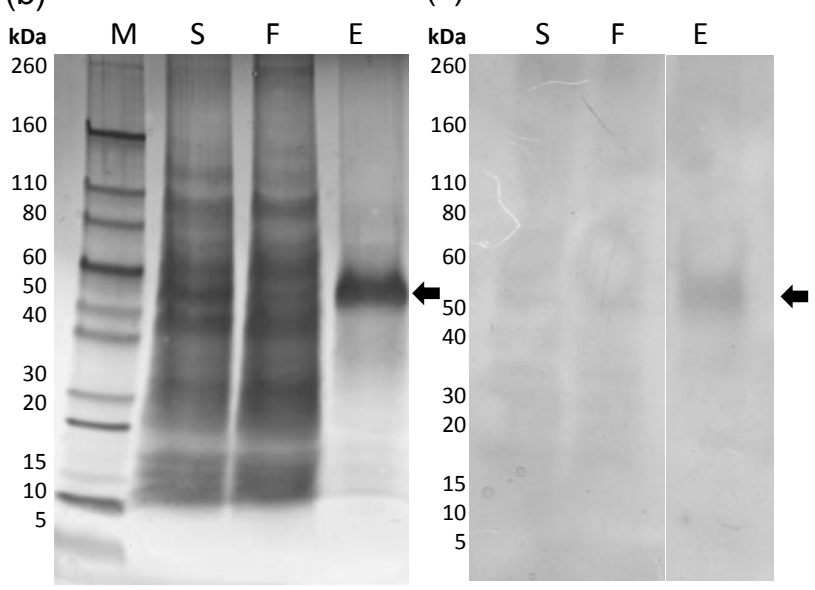

(d)

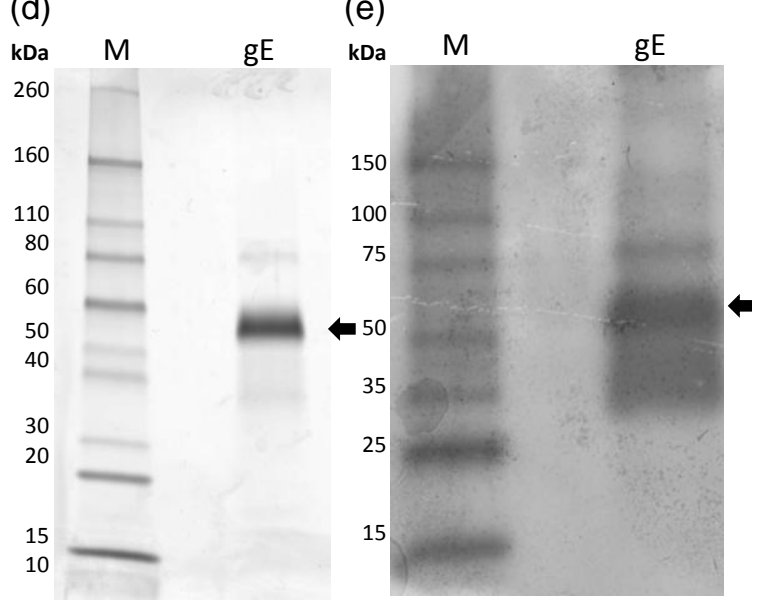

Fig. 1: Production, purification and characterization of BHV-1 glycoprotein E (gE) produced using Baculovirus expression systems. (a) Schematic representation of $\mathrm{gE}$ expression and purification workflow for FET and SPR sensor immobilization. (b) SDS-PAGE (4-15\% TGX precast gel with silver staining) analysis of ( $\mathrm{M}$ - molecular weight marker, $\mathrm{S}$ High Five supernatant, F - IMAC flow-through, and E - $250 \mathrm{mM}$ Imidazole elution). (c) Western blot using 1:500 goat-anti-BHV-1 primary antibody (S - High Five supernatant, F- IMAC flowthrough, and E - $250 \mathrm{mM}$ Imidazole elution). (d) SDS-PAGE and (e) western blot (using 1:500 goat-anti-BHV-1 primary antibody) of $\mathrm{gE}$ stocks prepared at $1 \mathrm{mg} / \mathrm{ml}$ for downstream sensor fabrication ( $\mathrm{M}$ - molecular weight marker, $\mathrm{gE}-\mathrm{gE}$ ). Arrow corresponds to $59 \mathrm{kDa} \mathrm{gE}$ protein. 

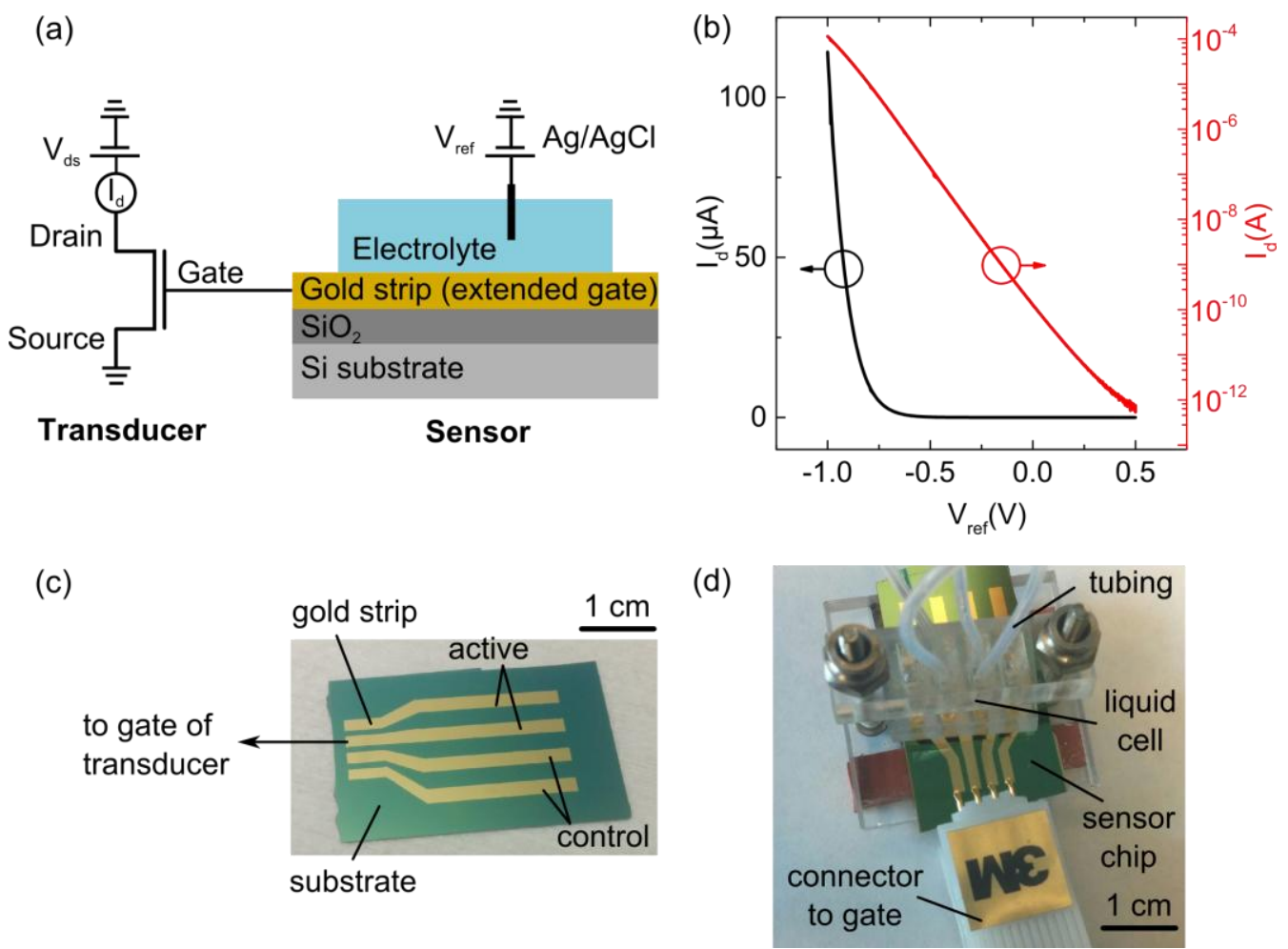

Fig. 2. EGFET sensor layout and characterization. (a) Schematic of the potentiometric sensing device, consisting of two parts: a sensor part for signal generation and a transducer part for signal amplification and readout. The active sensing surface is a thin gold film evaporated on a $\mathrm{Si} / \mathrm{SiO}_{2}$ substrate. The gold film is contact with the electrolyte and its surface potential depends on the amount of charged molecules attached to the surface. The gold surface can be chemically modified to achieve specific adsorption of certain biomolecules. To read out the potential changes on the gold surface, a commercial MOSFET is used as the readout transducer. The gold film acts as the extended gate of the MOSFET, and is electrically connected to the gate terminal. A reference electrode $(\mathrm{Ag} / \mathrm{AgCl})$ is immersed in the solution to gate the transistor through the liquid. Different solutions are delivered to the surface using microfluidics. (b) A typical transistor transfer curve showing that the drain current $I_{\mathrm{d}}$ can be modulated by sweeping the voltage at the

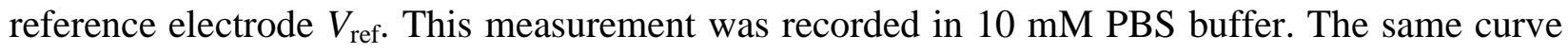
is shown on linear scale (left axis) and semi-log scale (right axis). (c) Image of a disposable sensing chip used in this study. Four $100 \mathrm{~nm}$ thick gold strips were e-beam evaporated through a shadow mask. Using a liquid cell shown in (d), two gold strips were functionalized with the protein $\mathrm{gE}$ (active), and the other two were functionalized with protein Hp (control). To read out the potential changes generated on the sensor surface, the individual gold strips were connected using a test clip shown in (d) to the gate of the transducer. 

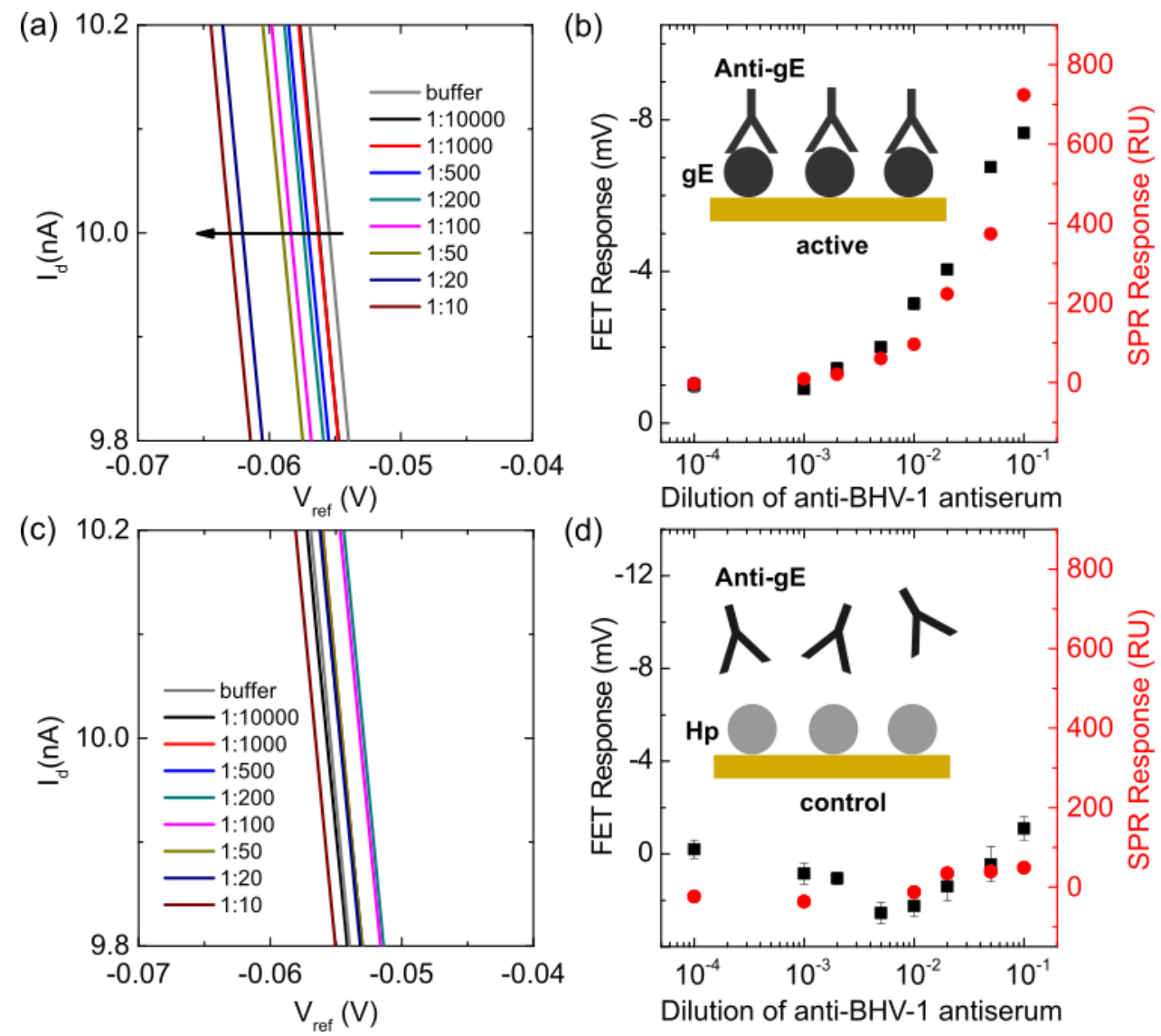

Fig. 3. Evaluation of EGFET and SPR performance for anti-BHV-1 antibody detection in serum. Two gold strips were functionalized differently and used as the active sensor (a-b) and the control (c-d). Both surfaces were measured in the same dilutions of antiserum. (a-b) The BHV-1 glycoprotein E ( $\mathrm{gE}$ ) was immobilized on the active gold strip using amine coupling, and acts as a capture antigen for anti-gE antibody present in antiserum (inset in b). (a) The transistor curves measured with the active gE-coated device shift to the left with increasing serum dilution in $10 \mathrm{mM}$ HPS-EP buffer. (b) The calibration curve in different antiserum dilutions. The FET response is defined as the voltage shift relative to the value in buffer (buffer FET response $=0$ $\mathrm{mV}$ ), measured at $I_{\mathrm{d}}=10 \mathrm{nA}$ (along the arrow in (a)). In principle, any other $I_{\mathrm{d}}$ value can be used as a reference, because the curves shift parallel to each other. The results are in excellent agreement with surface plasmon resonance (SPR) measurements (SAM chip, right axis). (c,d) The control sample was coated with the protein Haptoglobin (Hp) as a control for possible nonspecific binding. (c) The Hp-coated control does not show a clear trend with changing antiserum dilution. (d) The observed response due to non-specific binding is small compared to the specific response shown in (b). Again, the FET results agree very well with the SPR data (CM5 chip). 
(a)

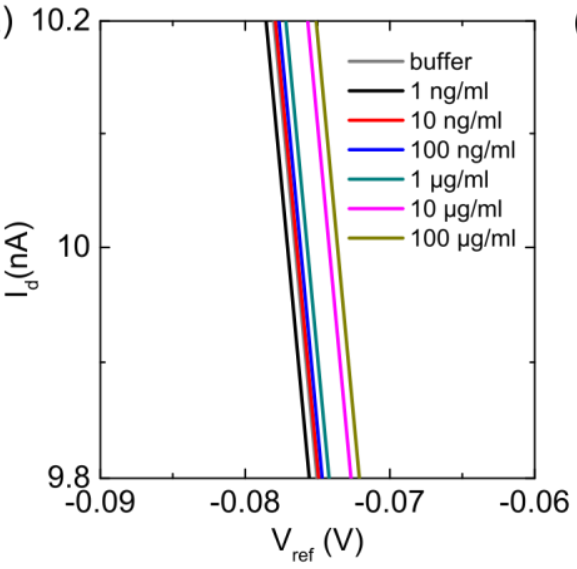

(b)

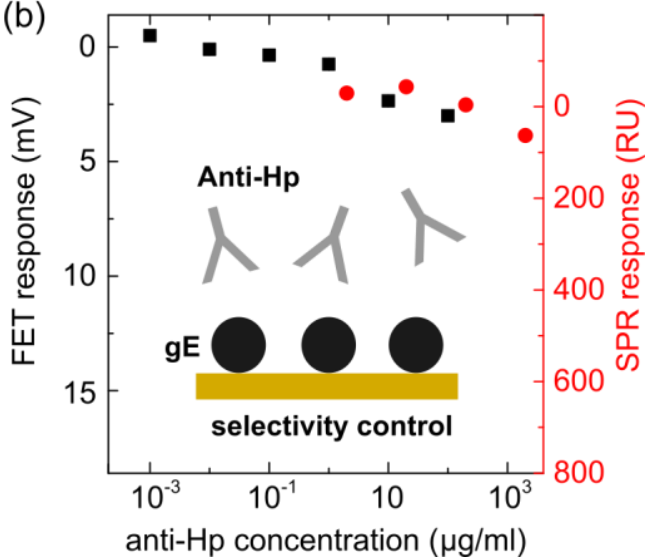

Fig. 4. Selectivity control of EGFET and SPR sensors immobilized with gE. (a) The active gE-coated sensor was measured in different concentrations of the haptoglobin antibody (anti-Hp) in $10 \mathrm{mM}$ HBS-EP buffer. At higher concentrations, a small shift to more positive values is observed, opposite to the response direction to anti-gE. The FET and SPR responses (CM5 chip) are compared in (b). The results suggest high selectivity of the gE-coated sensor toward anti-gE in solution.

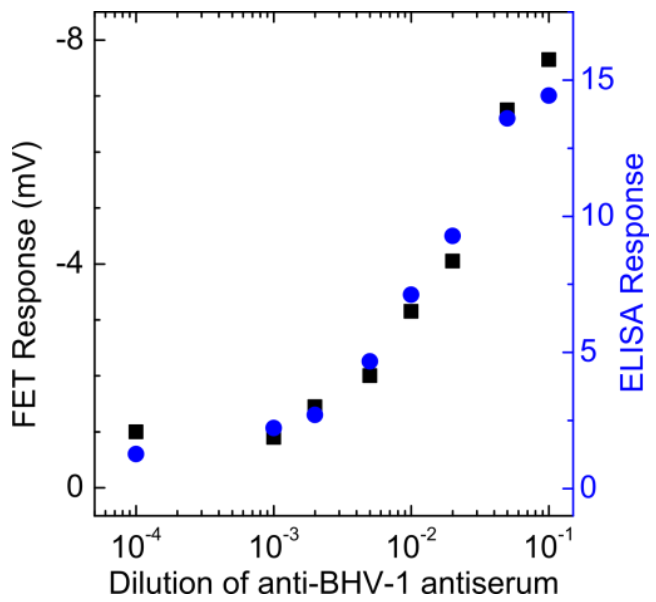

Fig. 5. Comparison of EGFET and ELISA for anti-BHV-1 antibody detection in serum. The response of a gE-coated FET sensor from Fig. 3b is plotted together with IDEXX gE blocking ELISA (1/absorbance) vs. the dilution of anti-BHV-1 antiserum. Comparable results (sensitivity and assay linearity) were obtained for both diagnostic platforms. 

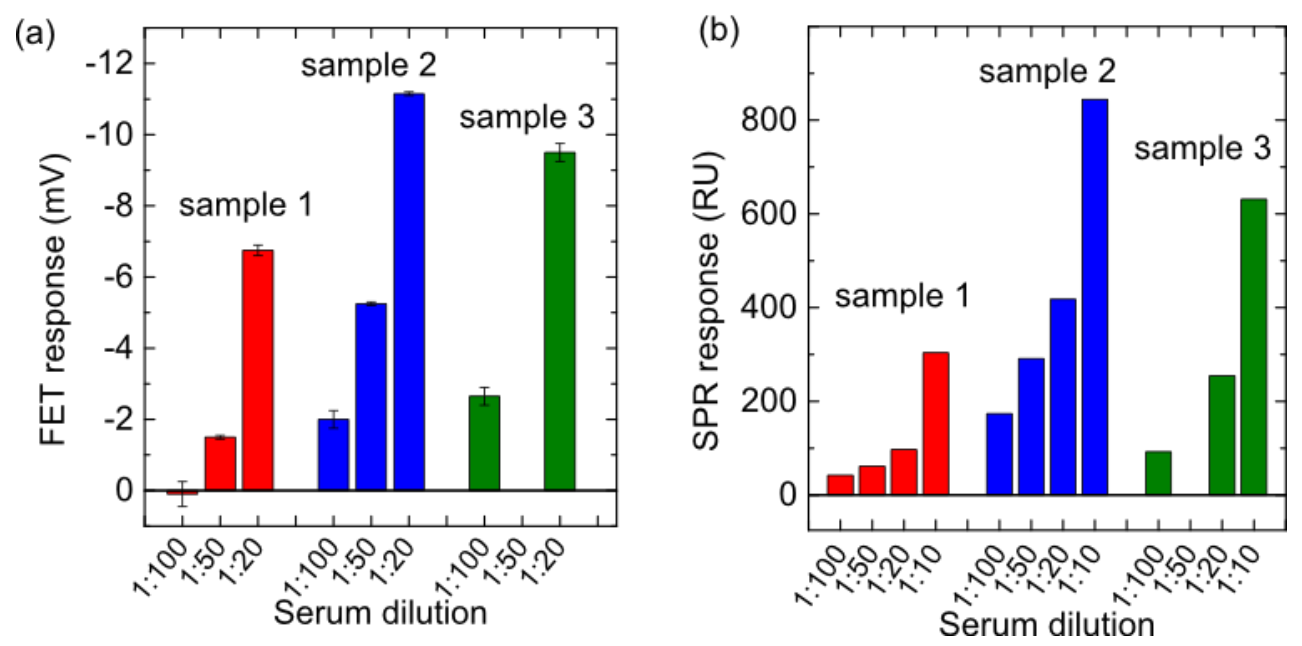

Fig. 6. Assessment of EGFET and SAM SPR performance for anti-BHV-1 detection in serum from BHV-1 infected animals. Sample 1 was from a BHV-1 negative animal, whereas samples 2 and 3 originated from BHV-1 positive animals. The measurements were performed in different dilutions of the same 3 blood samples. (a) The FET response is shown vs. the dilution of the 3 samples. The sensor response increases in higher dilutions, because more antibodies can bind to the surface. (b) SPR results, obtained with the same blood samples, show very similar behavior validating the FET response. In general, the response to sample $2>$ sample $3>$ sample 1, indicating different levels of antibodies in the serum. The data for the 1:50 dilution of sample 3 is missing due to an experimental error (no serum was added to the buffer). 


\section{References}

Anderson, N.L., Esquerblasco, R., Hofmann, J.P., Meheus, L., Raymackers, J., Steiner, S., Witzmann, F., Anderson, N.G., 1995. Electrophoresis 16 (10), 1977-1981.

Anderson, S., Wakeley, P., Wibberley, G., Webster, K., Sawyer, J., 2011. J. of Immunol. Methods 366 (12), 79-88.

Bedner, K., Guzenko, V.A., Tarasov, A., Wipf, M., Stoop, R.L., Just, D., Rigante, S., Fu, W., Minamisawa, R.A., David, C., 2013. Sensor Mater. 25 (8), 567-576.

Bedner, K., Guzenko, V.A., Tarasov, A., Wipf, M., Stoop, R.L., Rigante, S., Brunner, J., Fu, W., David, C., Calame, M., 2014. Sens. Actuators B 191, 270-275.

Bergveld, P., 2003. Sens. Actuators B 88 (1), 1-20.

Bernards, D.A., Owens, R.M., Malliaras, G.G., 2008. Organic semiconductors in sensor applications. Springer.

Brown, M., Faulkner, P., 1978. Appl. Environ. Microbiol. 36 (1), 31-35.

Campbell, P.M., Tarasov, A., Joiner, C.A., Ready, W.J., Vogel, E.M., 2015. ACS nano 9 (5), 5000-5008.

Chen, K.-I., Li, B.-R., Chen, Y.-T., 2011. Nano Today 6 (2), 131-154.

Chua, J.H., Chee, R.E., Agarwal, A., Wong, S.M., Zhang, G.J., 2009. Anal. Chem. 81 (15), 6266-6271.

Confer, A.W., 2009. Animal health research reviews / Conference of Research Workers in Animal Diseases 10 (2), 145-148.

Cui, Y., Wei, Q., Park, H., Lieber, C.M., 2001. Science 293 (5533), 1289-1292.

Dak, P., Nair, P., Go, J., Alam, M.A., 2013. Extended-gate biosensors achieve fluid stability with no loss in charge sensitivity. Device Research Conference (DRC), 2013 71st Annual, pp. 105-106. IEEE.

Donofrio, G., Bottarelli, E., Sandro, C., Flammini, C.F., 2006. Clin. Vaccine Immunol. 13 (6), 698-701.

El-Kholy, A.A., Abdou, E.R., Rady, D.I., Elseafy, M.M., 2013. J. Virol. Methods 191 (1), 33-40.

Ellis, J.A., 2009. Anim. Health Res. Rev. 10 (2), 149-153.

Fernandes, P.G., Seitz, O., Chapman, R.A., Stiegler, H.J., Wen, H.-C., Chabal, Y.J., Vogel, E.M., 2010. Appl. Phys. Lett. 97 (3), 034103.

Fu, W., Nef, C., Knopfmacher, O., Tarasov, A., Weiss, M., Calame, M., Schönenberger, C., 2011. Nano Lett. 11 (9), 3597-3600.

Fu, W., Nef, C., Tarasov, A., Wipf, M., Stoop, R.L., Knopfmacher, O., Weiss, M., Calame, M., Schoenenberger, C., 2013. Nanoscale 5 (24), 12104-12110.

Giovannini, S., Zanoni, M.G., Salogni, C., Cinotti, S., Alborali, G.L., 2013. Res. Vet. Sci. 95 (2), 576-579. Guan, W., Duan, X., Reed, M.A., 2014. Biosens. Bioelectron. 51, 225-231.

He, Q., Wu, S., Yin, Z., Zhang, H., 2012. Chem. Sci. 3 (6), 1764-1772.

Knopfmacher, O., Hammock, M.L., Appleton, A.L., Schwartz, G., Mei, J., Lei, T., Pei, J., Bao, Z., 2014. Nat. Commun. 5.

Knopfmacher, O., Tarasov, A., Fu, W., Wipf, M., Niesen, B., Calame, M., Schönenberger, C., 2010. Nano Lett. 10 (6), 2268-2274.

Knopfmacher, O., Tarasov, A., Wipf, M., Fu, W., Calame, M., Schönenberger, C., 2012. ChemPhysChem 13 (5), 1157-1160.

Lee, J., Dak, P., Lee, Y., Park, H., Choi, W., Alam, M.A., Kim, S., 2014. Sci. Rep. 4, 7352.

Lehman, D.J., Roof, L.L., Brideau, R.J., Aeed, P.A., Thomsen, D.R., Elhammer, A.P., Wathen, M.W., Homa, F.L., 1993. J. Gen. Virol. 74, 459-469.

Liao, C., Mak, C., Zhang, M., Chan, H.L., Yan, F., 2015. Adv. Mater. 27 (4), 676-681.

Lin, P., Luo, X., Hsing, I., Yan, F., 2011. Adv. Mater. 23 (35), 4035-4040.

Livi, P., Bedner, K., Tarasov, A., Wipf, M., Chen, Y., Schönenberger, C., Hierlemann, A., 2013. Sens. Actuators B 179, 293-300.

Mu, L., Chang, Y., Sawtelle, S.D., Wipf, M., Duan, X., Reed, M., 2015. Access, IEEE 3, 287-302.

Noor, M.O., Krull, U.J., 2014. Anal. Chim. Acta 825 (0), 1-25.

Raaperi, K., Orro, T., Viltrop, A., 2014. Vet. J. 201 (3), 249-256. 
Rajan, N.K., Duan, X., Reed, M.A., 2013. Wiley Interdiscip. Rev. Nanomed. Nanobiotechnol. 5 (6), 629645.

Roberts, M.E., Mannsfeld, S.C., Queraltó, N., Reese, C., Locklin, J., Knoll, W., Bao, Z., 2008. Proc. Natl. Acad. Sci. USA 105 (34), 12134-12139.

Rothberg, J.M., Hinz, W., Rearick, T.M., Schultz, J., Mileski, W., Davey, M., Leamon, J.H., Johnson, K., Milgrew, M.J., Edwards, M., Hoon, J., Simons, J.F., Marran, D., Myers, J.W., Davidson, J.F., Branting, A., Nobile, J.R., Puc, B.P., Light, D., Clark, T.A., Huber, M., Branciforte, J.T., Stoner, I.B., Cawley, S.E., Lyons, M., Fu, Y., Homer, N., Sedova, M., Miao, X., Reed, B., Sabina, J., Feierstein, E., Schorn, M., Alanjary, M., Dimalanta, E., Dressman, D., Kasinskas, R., Sokolsky, T., Fidanza, J.A., Namsaraev, E., McKernan, K.J., Williams, A., Roth, G.T., Bustillo, J., 2011. Nature 475 (7356), 348-352.

Sarkar, D., Liu, W., Xie, X., Anselmo, A.C., Mitragotri, S., Banerjee, K., 2014. ACS Nano 8 (4), $3992-$ 4003.

Sarkar, D., Xie, X., Liu, W., Cao, W., Kang, J., Gong, Y., Kraemer, S., Ajayan, P.M., Banerjee, K., 2015. Nature 526 (7571), 91-95.

Scott, P.R., 1994. Factors of Respiratory Disease: Review of Management Factors. In: Anthony, F.J. (Ed.), Bovine Respiratory Disease: Total Health Management, pp. 17-24. Schering-Plough Animal Health, Edinburgh, Scotland.

Snowder, G.D., Van Vleck, L.D., Cundiff, L.V., Bennett, G.L., Koohmaraie, M., Dikeman, M.E., 2007. J. Anim. Sci. 85 (8), 1885-1892.

Stern, E., Vacic, A., Rajan, N.K., Criscione, J.M., Park, J., Ilic, B.R., Mooney, D.J., Reed, M.A., Fahmy, T.M., 2010. Nat. Nanotechnol. 5 (2), 138-142.

Stoop, R.L., Wipf, M., Müller, S., Bedner, K., Wright, I.A., Martin, C.J., Constable, E.C., Fu, W., Tarasov, A., Calame, M., Schönenberger, C., 2015. Sens. Actuators B 220, 500-507.

Strube, W., Auer, S., Block, W., Heinen, E., Kretzdorn, D., Rodenbach, C., Schmeer, N., 1996. Vet. Microbiol. 53 (1-2), 181-189.

Tang, X., Bansaruntip, S., Nakayama, N., Yenilmez, E., Chang, Y.-l., Wang, Q., 2006. Nano Lett. 6 (8), 1632-1636.

Tarasov, A., Campbell, P.M., Tsai, M.-Y., Hesabi, Z.R., Feirer, J., Graham, S., Ready, W.J., Vogel, E.M., 2014. Adv. Funct. Mater. 24, 6389-6400.

Tarasov, A., Fu, W., Knopfmacher, O., Brunner, J., Calame, M., Schönenberger, C., 2011. Appl. Phys. Lett. 98 (1), 012114.

Tarasov, A., Tsai, M.-Y., Flynn, E.M., Joiner, C.A., Taylor, R.C., Vogel, E.M., 2015a. 2D Mater. 2 (4), 044008 .

Tarasov, A., Wipf, M., Bedner, K., Kurz, J., Fu, W., Guzenko, V.A., Knopfmacher, O., Stoop, R.L., Calame, M., Schönenberger, C., 2012a. Langmuir 28 (25), 9899-9905.

Tarasov, A., Wipf, M., Stoop, R.L., Bedner, K., Fu, W., Guzenko, V.A., Knopfmacher, O., Calame, M., Schönenberger, C., 2012b. ACS Nano 6 (10), 9291-9298.

Tarasov, A., Zhang, S., Tsai, M.-Y., Campbell, P.M., Graham, S., Barlow, S., Marder, S.R., Vogel, E.M., 2015b. Adv. Mater. 27 (7), 1175-1181.

Thiry, E., Saliki, J., Bublot, M., Pastoret, P.P., 1987. Comp Immunol Microbiol Infect Dis 10 (1), 59-63.

Thompson, P.N., Stone, A., Schultheiss, W.A., 2006. J. Anim. Sci. 84 (2), 488-498.

Tsai, M.-Y., Tarasov, A., Hesabi, Z.R., Taghinejad, H., Campbell, P.M., Joiner, C., Adibi, A., Vogel, E.M., 2015. ACS Appl. Mater. Interfaces 7 (23), 12850-12855.

Wellenberg, G.J., Verstraten, E.R., Mars, M.H., Van Oirschot, J.T., 1998. J. Clin. Microbiol. 36 (2), 409413.

Wipf, M., Stoop, R.L., Tarasov, A., Bedner, K., Fu, W., Wright, I.A., Martin, C.J., Constable, E.C., Calame, M., Schönenberger, C., 2013. ACS Nano 7 (7), 5978-5983.

Zhan, B., Li, C., Yang, J., Jenkins, G., Huang, W., Dong, X., 2014. Small 10 (20), 4042-4065. 